edge is blackish on the Sth and 9th segments, the vulvar lamina with the lobes more pointed on the tip, and better separated at the base.

If $D$. Alavicosta, as I believe, is merely a variety of $D$. madida, four smaller females from Victoria, Vancouver Island, July, can not be separated. The smallest is $3 \mathrm{Imm}$. in length ; alar expanse 5omm.; pterostig- ma $2 \mathrm{~mm}$. The wings are fumose, the anterior margin flavescent in one, another has the base to the nodus or only beyond the triangle flavescent; the anterior pale band on the sides of the thorax is plainly visible; a large black band on each side of the abdomen is complete; in one female the black extends from the end of the segments to the middle ridge.

\title{
SYNONYMY OF THE HOMOPTERA DESCRIBED BY SAY, HARRIS AND FITCH.
}

\author{
BY E. P. VAN DUZEE, BUFFALO, N. Y.
}

In the homoptera as in other branches of our favorite science a solid foundation was laid by that illustrious pioneer of American entomology, Thomas Say, whose brief but concise descriptions of many of our native insects have elicited universal commendation from later students. Scarcely inferior to that of Say is the work done many years later by Asa Fitch most of whose numerous species may be readily recognized from his short, clear diagnoses. Intermediate in point of time comes the work of Thaddeus William Harris to whom, however, we owe the description of but very few homopterous insects. In the present paper I propose to give the corrected nomenclature of the homoptera cicadinae described by these honored leaders of American entomology so far as it is known to myself or has been made known by recent writers.
Many of these names have been in common use for years, but a number are to be found only in rare or little known papers, and not a few are now for the first time systematically placed. In a few cases where I still feel in doubt the reference is followed by a question point.

Of the $7 \mathrm{r}$ species described by Say 60 are known to me; all of those described by Harris, 6 in number, and all but 15 of the 74 species described by Fitch. Two of those described by Say, viz., Fassus sanctus and Membracis subulata, are, so far as I can learn, unknown to our later entomologists. In the cicadidae and typhlocybidae I have quoted all references from the studies of Messrs. Uhler and Woodworth whose valuable synonymical notes on these families may be found in Ent. Amer. v. 4, 2I and 8I, and Psyche, 
v. 5, p. 67 and $2 \mathrm{II}$. In these cases as in others where I have not personally studied the insect I have appended my authority for the name employed. To species still unknown to me in nature I have prefixed an asterisk.

The following species require no change of name to bring them into correspondence with our modern nomenclature.

Described by SAY :

Cicada dorsata.

* "6 marginata.

* 6. vitripennis.

Aphrophora 4-notata.

Tettigonia bifida.

66 hieroglyphica.

\section{Described by Fitch :}

* Anotia burnetii.

* 66 robertsonii.

* 66 westwoodi.

Bruchomorpha dorsata.

Ceresa brevicornis.

66 taurina.

* Cicada superba (auct. Woodworth).

* Cixius cinctifrons (auct. Ashmead).

* Cixius coloepium (auct. Ashmead).

Cixius pini.

Clastoptera proteus.

* 66 pini (auct. Ashmead).

* Clastoptera testacea (auct. Ashmead).

* Cyrtosia fenestrata (auct. Uhler). Eucanthus orbitalis.

Gypona flavilineata.

66 scarlatina.

Helochara communis.
Idiocerus alternatus?

6 lachrymalis.

66 maculipennis.

6 pallidus.

6 suturalis.

* Naso robertsoni (auct. Uhler).

Otiocerus amyoti.

* 66 kirbyi.

6 signoreti.

Pediopsis trimaculata.

6 viridis.

Penthimia americana.

Telamona concava.

"6 coryli.

6 fasciata.

66 reclivata.

Tettigonia tripunctata.

Thelia crataegi.

Some of them will doubtless prove on further study to be but varieties of other forms and a few may. yet have to be placed as synonyms of earlier described species.

The following names required to be changed.

Described by SAY :

Aphrophora bilineata $=$ Philaenus lineatus Linn.

Cercopis bicincta $=$ Monecphora id.

Cercopis obtusa = Clastopera id.

Cercopis parallela $=$ Aphrophora id.

Cercopis 4 -angularis = Lepyronia id.

* Cicada aurifera = Cicada marginata Say (auct. Woodworth).

Cicada hieroglyphica $=$ Tettigea $\mathrm{id}$, (auct. Uhler).

Cicada parvula $=$ Carineta id. (auct. Uhler).

Cicada pruinosa $=$ Cicada tibicen 
Linn. (auct. Woodworth), probably a variety.

Cicada rimosa $=$ Tibicen id. (auct. Woodworth).

Cicada synodica $=$ Tibicen id. (auct. Woodworth).

Delphax tricarinata.

No genus yet established seems properly to include this species.

Flata bivittata = Amphiscepa id.

Flata bullata $=$ Thionea id.

* Flata conica.

This name is not changed by $\mathrm{Mr}$. Ashmead. (See Smith's Cat. Ins. of New Jersey, p. 438.)

Flata humilis = Oliarus id.

Flata nava $=$ Phypia (?) id.

* Flata opaca = Helicoptera id. (auct. Uhler).

Flata pallida $=$ Helicoptera id.

Flata pruinosa $=$ Ormenis id.

Flata 5 -lineata $=$ Oliaris id.

Flata stigmata $=$ Cixius id.

Fulgora sulcipes $=$ Scolops id.

Jassus acutus $=$ Platymetopius id.

Jassus clitellarius $=$ Thamnotettix id.

Jassus immistus $=$ Scaphoideus id.

Jassus inimicus $=$ Deltocephalus id.

Jassus irroratus $=$ Phlepsius id.

Jassus novellus $=$ Agallia id .

Jassus olitorius $=$ Jassus (as restricted by Stål) icl.

Jassus seminudus $=$ Athysanus id.

Jassus subbifasciatus = Jassus olitorius Say 9 .

* Jassus verticis.

$\mathrm{Mr}$. Uhler places this as congeneric with Idiocerus pallidus $\mathrm{Fh}$. and ramentosus Uh. Possibly it will prove identical with Idiocerus alternatus Fh. which name it would then supercede.

Membracis arquata.

This is congeneric with an undescribed form placed by $\mathrm{Mr}$. Uhler in Ophiderma Fairm., but the transverse apical cell of the elytra will perhaps entitle them to generic distinction.

Membracis belligera $=$ Platycotis id.

Membracis binotata = Enchenopa id.

Membracis calva $=$ Acutalis id.

Membracis concava $=$ Publilia id.

Membracis diceros = Ceresa id.

Membracis festina $=$ Stictocephala id.

Membracis goniphora $=$ Stictocephala inermis Fab.

Membracis inornata = Atymna id.

Membracis latipes = Campylenchia curvata Fab.

Membracis marmorata $=$ Carynota id.

Membracis mera $=$ Carynota id.

Membracis 4-vitlata = Platycotis id.

Membracis semicrema $=$ Acutalis id.

* Membracis tartarea $=$ Acutalis id. (auct. Uhler).

Membracis trilineata $=$ Cyrtosia id.

This may prove to be a synonym of

C. mutica Fab.

Membracis vau $=$ Cyrtosia id.

Tettigonia basilaris = Typhlocyba id. (auct. Woodw.)

* Tettigonia coagulata = Homalodisca id. (auct. Stål).

Tettigonia comes $=$ Typhlocyba id. (auct. Woodw.)

Tettigonia limbata $=$ Oncometopia id. 
Tettigonia mixta $=$ Acocephalus id.

Tettigonia mollipes = Diellrocephala id.

Tettigonia obliqua $=$ Typhlocyba id. (auct. Woodw.)

* Tettigonia occatoria.

Dr. Stål does not change this combined name in his Hemip. Mexicana.

Tettigonia octolineata = Gypona id.

Tettigonia 4 -vittata $=$ Diedrocephala coccinea Forst.

Tettigonia trifasciata $=$ Typhlocyba id. (auct. Woodw.)

* Tettigonia versuta $=$ Diedrocephala id. (auct. Woodw.)

\section{Described by Fitch :}

Acocephalus vitellinus=Selenocephalus id (auct. Ashmead).

Amblycephalus curtisii $=$ Athysanus id.

Amblycephalus melsheimeri $=$ Deltocephalus id.

Amblycephalus sayi $=$ Deltocephalus id.

*Aphrophora signoreti.

Doubtless correct.

Athysanus abietis=Bythoscopus variabilis Fh. $\delta$.

*Athysanus fagi.

Certainly a species of Bythoscopus.

Athysanus fenestratus=Bythoscopus id.

Athysanus minor=Bythoscopus id.

Athysanus nigrinasi $=$ Bythoscopus id.

Athysanus variabilis $=$ Bythoscopus id.

Aulacizes novaeboracensis $=$ Diedrocephala id.
Bythoscopus strobi=Phlepsius id.

Bythoscopus tergatus $=$ Grypotes id. (auct. Uhler).

Bythoscopus unicolor $=$ Grypotes id. (auct. Uhler).

Cicada robertsoni $=$ Cicada dorsata Say. (auct. Woodw.)

Cixius impunctatus $=$ Myndus id.

Delphax arvensis=Liburnia id.

Delphax dorsalis $=$ Stenocranus id.

*Empoa coccinea $=$ Typhlocyba id. (auct. Woodw.)

Empoa querci $=$ Typhlocyba id. (auct. Woodw.)

Erythroneura affinis=Typhlocyba id. (auct. Woodw.)

Erythroneura tricincta $=$ Typhlocyba id. (auct. Woodw.)

Erythroneura vitifex=Typhlocyba id. (auct. Woodw.)

Erythroneura vulnerata $=$ Typhlocyba id. (auct. Woodw.)

Jassus fulvidorsum $=$ Phlepsius id.

Lepyroria saratogensis=A phrophora id.

* Monecphora ignipecta $=$ var. of Monecphora bicincta Say.

Poeciloptera vulgaris=Lamenia id.

Smilia auriculata $=$ Archasia galeata Fab.

Smilea castaneae $=$ Atymna inornata Say $\delta(?)$.

(See under Telamona unicolor)

Smilia querci=Atymna id.

Telamona fagi $=$ Heliria scalaris Fairm.

Telamona querci $=$ Telamona monticola Fab.

Telamona tristis $=$ Telamona coryli Fh. . 
These forms I have taken in coitu. I have never met with a $\hat{o}$ tristis nor a o coryli.

Telamona unicolor $=$ Telamona fasciata Fitch. \& (?).

These forms always occur together and of the large number I have examined the former are always females and the latter males. On the strength of this I have placed them as a single species and am confident that future observations will justify me in so doing. The case is the same with Smilia castaneae Fitch. and Membracis inornata Say. ${ }^{1}$

Tragopa dorsalis $=$ Acutalis id.

Uroxiphus caryæ $=$ Microcentrus id.

\section{Described by HaRris:}

Cicada canicularis $=$ var. of Cicada tibicen Linn. (auct. Woodw.)

Membracis ampelopsidis=Telamona id.

\footnotetext{
1 The following is the correct synonymy of this genus so far as I can make out :

Telamona reclivata $\mathrm{Fitch}$.

monticola Fab.

querci Fitch.

ampelopsidis Harr.

cissi Harr. (List.) cyrtops Fairm.

concava Fitch.

ornata Emmons.

coryli Fitch $\sigma^{x}$. tristis Fitch 9 .

fasciata Fitch 3 . unicolor Fitch $q$.

*mexicana Stål.

*excelsa Fairm.

*pyramidata Uhler.
}

Membracis univittata $=$ Thelia id.

Tettigonia vitis $=$ Typhlocyba id. (auct. Woodw.)

Tettigonia rosæ $=$ Typhlocyba id. (auct. Woodw.)

Tettigonia fabæ $=$ Typhlocyba id. (auct. Woodw.)

The Centrotus acuminatus of Fabricius has been placed by Say, Harris and others as the $q$ of his Membracis bimaculata. I have however both sexes of both of these species; the females scarcely differ from the males in form. Our described species of this genus are.

Thelia bimaculata Fab. uhleri Stål. *turriculata Emmons. crataegi Fitch. univittata Harr. acuminata Fab.

Carynota Fitch. Dr. Stål is mistaken in placing this genus as a synonym of Ophiderma Fairm. It is quite widely separated from that genus but is very near his Optilete of which Thelia porphyrea Fairm., a species unknown to me, is the type. It is possible that Stål was unacquainted with the Membracis mera Say, which is properly the type of Fitch's genus, and hence was deceived by Dr. Fitch's erroneous reference of Membracis arquata Say to his Carynota. 

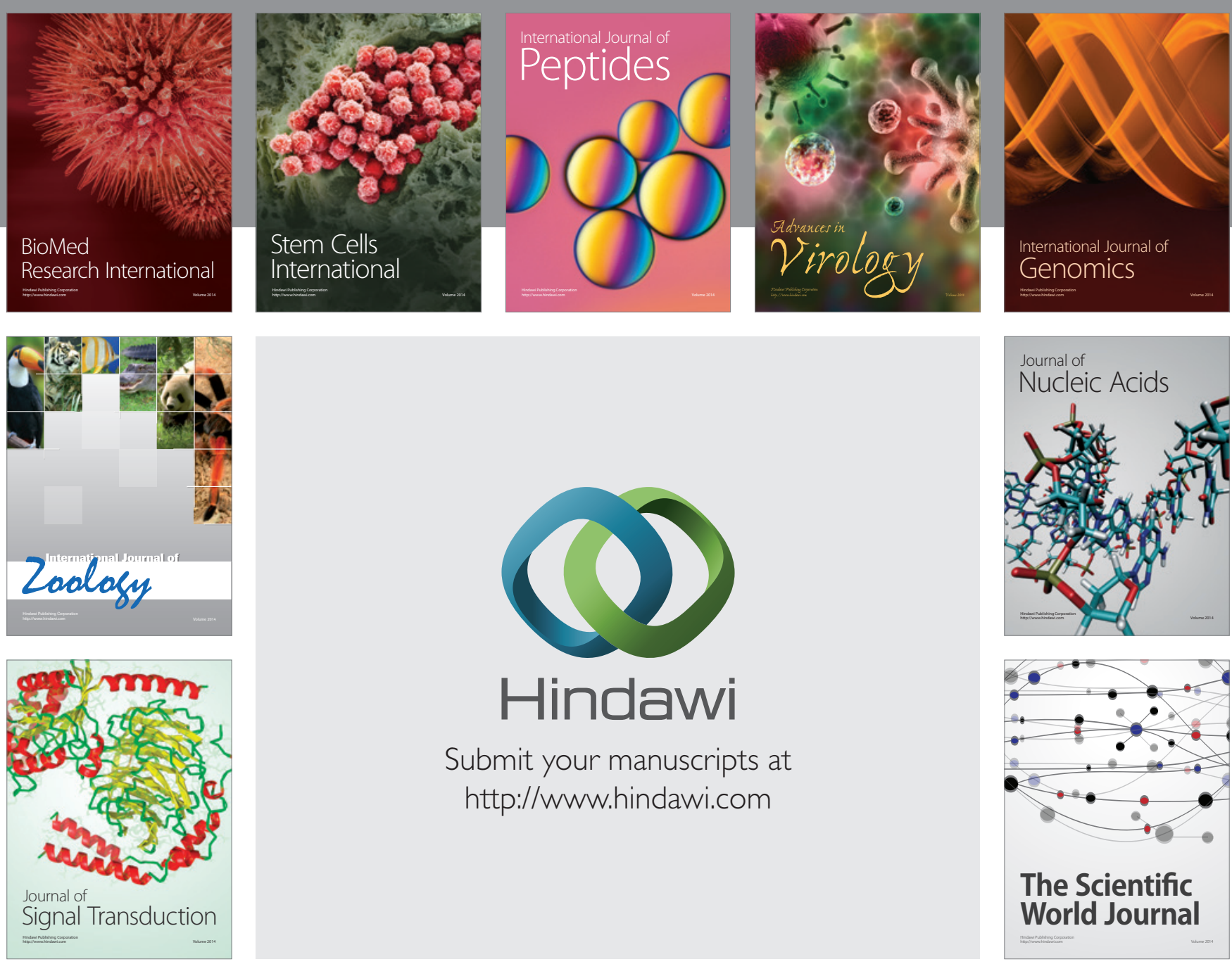

Submit your manuscripts at

http://www.hindawi.com
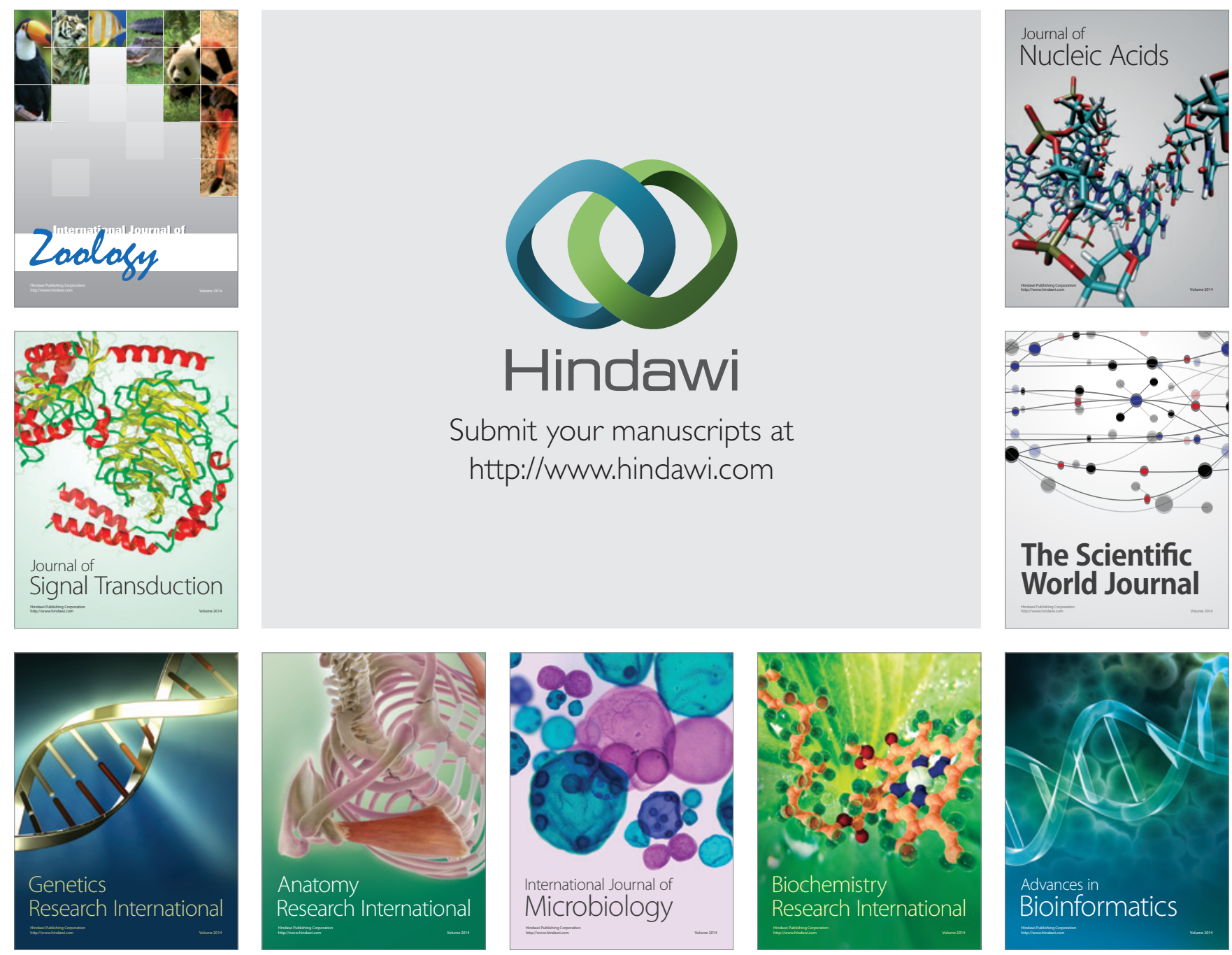

The Scientific World Journal
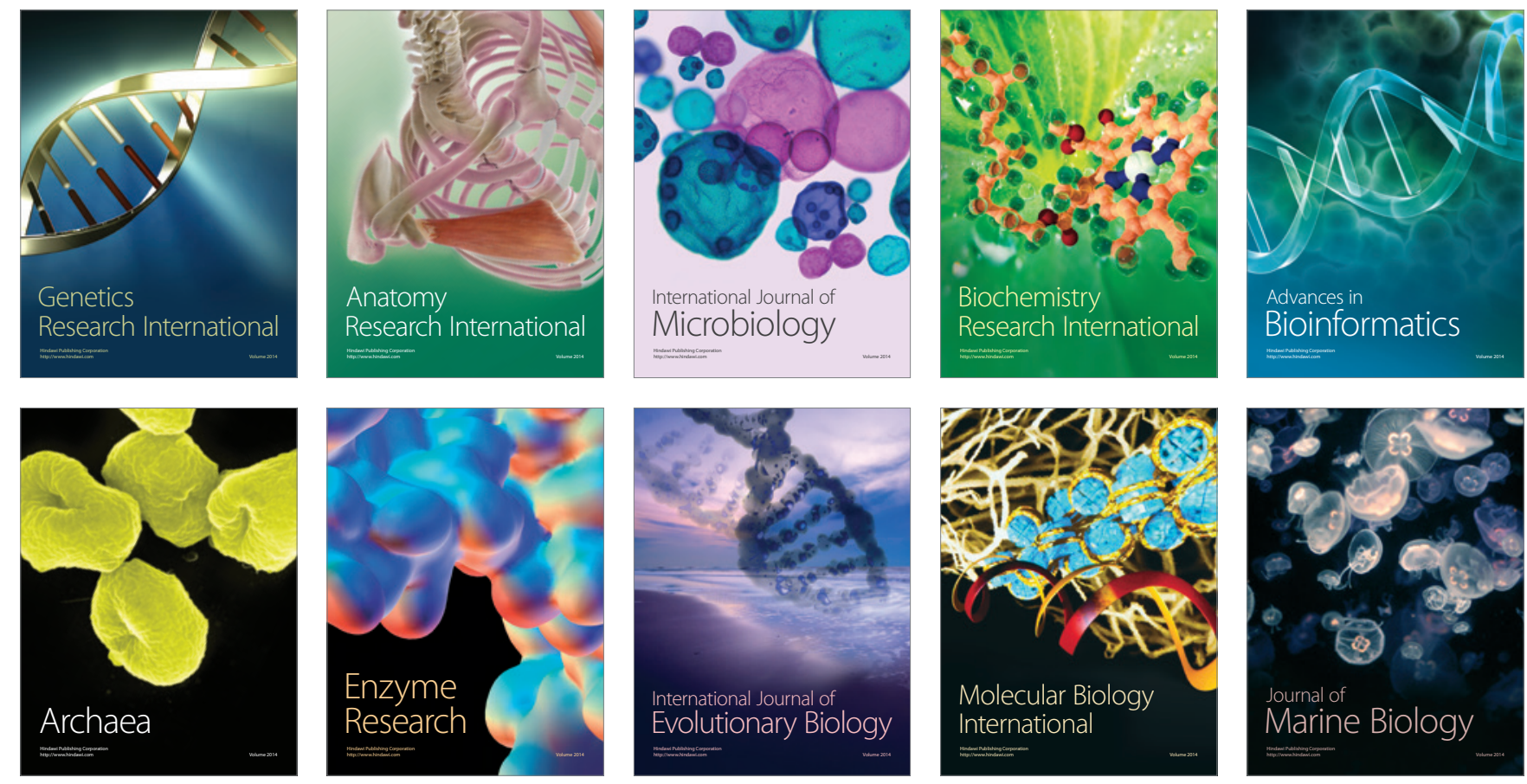